\title{
Stagnation Point Nonequilibrium Radiative Heating and the Influence of Energy Exchange Models
}

\author{
Lin C. Hartung, ${ }^{*}$ Robert A. Mitcheltree, and Peter A. Gnoffo ${ }^{\dagger}$ \\ NASA Langley Research Center, Hampton, VA 23665-5225
}

\begin{abstract}
A nonequilibrium radiative heating prediction method has been used to evaluate several energy exchange models used in nonequilibrium computational fluid dynamics methods. The radiative heating measurements from the FIRE II flight experiment supply an experimental benchmark against which different formulations for these exchange models can be judged. The models which predict the lowest radiative heating are found to give the best agreement with the flight data. Examination of the spectral distribution of radiation indicates that despite close agreement of the total radiation, many of the models examined predict excessive molecular radiation. It is suggested that a study of the nonequilibrium chemical kinetics may lead to a correction for this problem.
\end{abstract}

\section{Introduction}

Many proposed missions envision the use of aerobraking to return payloads from geostationary orbit, the Moon, and Mars. To design vehicles for this purpose, knowledge of the

*Aerospace Engineer, Aerothermodynamics Branch. Member AIAA.

$\dagger$ Aerospace Engineer, Aerothermodynamics Branch. Associate Fellow AIAA.

Presented as Paper 91-0571 at the AIAA 29th Aerospace Sciences Meeting, Reno, NV, Jan. 7-10, 1991; received Jan. 28, 1991; revision received June 17, 1991; accepted for publication June 17, 1991. Copyright (c)1991 by the American Institute of Aeronautics and Astronautics, Inc. No copyright is asserted in the United States under Title 17, U.S. Code. The U.S Government has a royalty-free license to exercise all rights under the copyright claimed herein for Governmental purposes. All other rights are reserved by the copyright owner. 
total heating environment is required. The radiative heat load on a vehicle moving through the atmosphere increases as the velocity and size increase, so that many of these missions will experience significant radiative heating. Accurate prediction of radiative as well as convective heating therefore becomes important to designers and mission planners. Since these vehicles will spend a large portion of their atmospheric trajectory at high altitude, where low freestream density can lead to significant thermal and chemical nonequilibrium effects, the tools used to predict the heating environment must account for nonequilibrium. Radiative heating predictions therefore depend on flowfields obtained from nonequilibrium computational fluid dynamics (CFD) codes, like LAURA (the Langley Aerothermodynamic Upwind Relaxation Algorithm ${ }^{1}$ ). These flowfield solvers contain energy exchange models that have yet to be validated. While perturbations in these models may have little effect on convective heating, their effect on the radiative heating level can be large. Experimental data on this question are limited, consisting almost exclusively of the FIRE flights of the mid-1960's. ${ }^{2}$

The objective of this study is to examine a number of the energy exchange models implemented in LAURA for their influence on stagnation point nonequilibrium radiative heating. By comparison with the FIRE II flight radiometer data, some insight into the validity of the various forms proposed for these exchange models may be obtained.

LAURA is a nonequilibrium CFD code that uses Park's two-temperature approximation, ${ }^{3}$ in which the heavy-particle-translational/rotational energy mode (hereafter referred to as the translational mode), and the vibrational/electronic/electron-translational energy mode (referred to as the vibrational mode ${ }^{4}$ ) are considered separately. LAURA contains a number of semi-empirical models governing the exchange of energy between these modes.

The radiation model used in this study is the Langley Optimized Radiative Nonequilibrium (LORAN) code, which is a recent development. ${ }^{5}$ It treats the radiation spectrum in moderate detail, accounting for all mechanisms of importance. In this paper, only the subrange of the visible and infrared spectrum where the FIRE radiometer windows are transparent is included. Gas absorption is negligible in this spectral region so the radiative transport 
is calculated assuming an optically thin shock layer. The tangent slab approximation is also invoked since the FIRE forebody is blunt.

The FIRE flight experiment was conducted during the 1960's to determine the heat load on an Apollo-type vehicle entering Earth's atmosphere at $11.4 \mathrm{~km} / \mathrm{sec}^{2,6}$ Of the two flights, the first experienced telemetry problems that made data reduction and analysis difficult as well as control problems during the second half of the entry that resulted in substandard data. These problems were corrected before the second flight, which provided good measurements of the total and spectral radiation at the stagnation point during the 40 second entry period. ${ }^{7,8}$ The second flight will be emphasized here.

Solutions are obtained with the LAURA code at three points in the FIRE II trajectory that span the range from extreme nonequilibrium early in the flight to a near-equilibrium point approaching peak heating. Each point is solved using the different energy exchange models under consideration. The flowfield conditions are then input to the LORAN code to obtain predictions of the radiative heating. The results are compared with the flight radiometer data to evaluate the validity of the various energy exchange models.

\section{Theory}

The LAURA code contains a number of semi-empirical energy exchange models that govern the transfer of energy between the translational and vibrational modes under nonequilibrium conditions. Several of these models continue to be the subject of debate ${ }^{4}$ as there has been no definitive determination of their validity because of a lack of experimental or flight

data. This study will examine the effect of models for the rate controlling temperature for dissociation, vibrational relaxation cross section, and energy exchange during dissociation. The effect of chemical kinetics is briefly examined as well. Energy exchange during electron impact ionization was considered, but since it has little effect under the low ionization levels of the FIRE flights, it was not pursued. 


\section{Dissociation Temperature}

The dissociation temperature, $T_{d}$, is the rate-controlling temperature for dissociation reactions. Several formulations have been proposed for this temperature, notably by Park and his co-workers. ${ }^{3,9}$ These models are empirical and consist of a geometric weighting of the translational temperature $T_{t}$ and the vibrational temperature $T_{v}$ as follows:

$$
T_{d}=T_{t}^{m} T_{v}^{n}
$$

The choice of powers $m$ and $n$ adjusts the weight given to each temperature. The recent trend has been to increase the weighting of the translational temperature $T_{t}$ because of indications that heavy particle collisions are more important than other mechanisms in causing dissociation. This study considers three different models with $(m, n)$ equal to $(.5, .5),(.7, .3)$, and $(1,0)$. The first set is the original Park two-temperature proposal, the second the more recent model, and the third is included to study the extreme case of dissociation controlled by the heavy particle temperature alone.

For convenience in identifying the various cases in this paper, a run identifier is used. The dissociation temperature models will be denoted by 55, 79, and 10, respectively, in the third and fourth digits of this identifier.

\section{Vibrational-Translational Energy Exchange Cross Section}

The equilibration of the vibrational and translational energy modes is modelled using a relaxation time, $\tau_{v}$, for each species. Millikan and White ${ }^{10}$ proposed a semi-empirical formulation for $\tau_{v}^{M W}$ in the range of 300 to $8000 \mathrm{~K}$. Park ${ }^{11}$ suggests an additional collision limiting correction, $\tau_{v}^{P}$, for high temperatures where the Millikan-White correlation predicts excessively fast relaxation. The total relaxation time is then

$$
\tau_{v}=\tau_{v}^{M W}+\tau_{v}^{P}
$$

where Park's contribution is

$$
\tau_{v}^{P}=\left(\sigma_{v} \overline{c_{s}} n_{s}\right)^{-1}
$$


In this expression $\overline{c_{s}}$ is the average velocity and $n_{s}$ the number density of molecular species $s$, and $\sigma_{v}$ is an effective cross section for vibrational relaxation. The latter quantity has been the subject of some debate, with three different values receiving support in various efforts to match the limited experimental data that has bearing on the question:

$$
\begin{gathered}
\sigma_{v}=10^{-21}\left(50,000 / T_{t}\right)^{2} \mathrm{~m}^{2} \\
\sigma_{v}=10^{-20} \mathrm{~m}^{2} \\
\sigma_{v}=10^{-21} \mathrm{~m}^{2}
\end{gathered}
$$

Equation 5 is the original proposal, which assumes this cross section to be one tenth of the elastic cross section. Equation 6 was suggested when Eq. 5 seemed still too high. Equation 4 is a modification of Eq. 6 that reduces the contribution of $\tau_{v}^{P}$ at low temperatures. All three models are considered in this study, and are identified by the notation $v 1$, v2, and v3, respectively.

A further modification to this energy exchange process has been proposed by Park. ${ }^{3}$ He argues that vibrational relaxation exhibits a diffusion-like behavior at high temperatures which requires a correction to the relaxation time. However, this modification requires evaluation of the post-shock levels of $T_{t}$ and $T_{v}$. Interpretation of these quantities in a shock capturing solution is somewhat ambiguous. Improper definition can lead to instabilities in some circumstances, consequently no attempt was made to include this modification.

\section{Energy Exchange in Dissociation}

When a diatomic molecule dissociates, the vibrational energy it contained is consumed by the higher ground states (heats of formation) of the constitutive atoms. The energy thus removed must be accounted for in the vibrational energy equation. The amount of energy lost is commonly assumed to be the average vibrational energy at the local conditions, $\overline{e_{v}}$. Because dissociation from a higher vibrational state may be more probable (the concept of preferential dissociation), several other formulations have been proposed. ${ }^{4}$ 
One model assumes that the vibrational energy loss in dissociation, $\Delta E_{v}$, is some fraction of the dissociation energy of a molecule measured from its ground state, $D$ :

$$
\Delta E_{v}=c_{1} D \quad\left(0<c_{1} \leq 1\right)
$$

Sharma et al. ${ }^{9}$ have proposed using $c_{1}=0.3$. This model was considered, but since it predicts the non-physical result of a negative $T_{v}$ in the shock region, it was not pursued.

Park $^{12}$ has proposed that

$$
\Delta E_{v}=D-k T_{t}
$$

where $\mathrm{k}$ is Boltzmann's constant, so that the energy lost is the dissociation energy minus the average translational energy. This model arises from the assumption that dissociation only occurs from levels that are within the average collisional energy of the dissociation threshold (similar to the mechanism of bound-free radiation). Again, this $\Delta E_{v}$ is too large and results in an unrealistically small $T_{v}$ behind the shock.

A third proposed model assumes that dissociation occurs from some vibrational state(s) above the average. This is expressed as:

$$
\Delta E_{v}=c_{2} \overline{e_{v}} \quad\left(c_{2}>1 .\right)
$$

The best value of $c_{2}$ to use in this empirical model is unknown, and may depend on $\overline{e_{v}}$ (or $T_{v}$ ). In this work $c_{2}=2$ has been selected to provide an initial assessment of the model. The suffix Devs is used in the run identifier for these cases.

\section{FIRE II Flight Experiment}

The FIRE flight vehicle was an Apollo-like geometry with a layered beryllium heatshield. (The second layer had a geometry identical to Apollo.) Each of the three layers was used up to a temperature limit and jettisoned, resulting in three periods of prime data during the entry. The first heatshield had a nose radius of $0.935 \mathrm{~m}$ and a diameter of $0.672 \mathrm{~m}$. All the cases considered here are for this first heatshield, which was jettisoned near the point of 
Table 1: Selected FIRE II Trajectory Conditions

\begin{tabular}{cccccc}
\hline \hline Case & Time & Altitude & Velocity & Density & $T_{w}$ \\
& $(\mathrm{sec})$ & $(\mathrm{km})$ & $(\mathrm{km} / \mathrm{sec})$ & $\left(\mathrm{kg} / \mathrm{m}^{3}\right)$ & $(\mathrm{K})$ \\
\hline $\mathrm{A}$ & 1631 & 84.59 & 11.37 & $9.15 \mathrm{e}-6$ & 460 \\
$\mathrm{~B}$ & 1634 & 76.41 & 11.36 & $3.72 \mathrm{e}-5$ & 615 \\
$\mathrm{C}$ & 1637.5 & 67.04 & 11.25 & $1.47 \mathrm{e}-4$ & 1030 \\
\hline \hline
\end{tabular}

peak heating. For this study it has been modelled as a sphere with an effective nose radius of $0.747 \mathrm{~m}$. A fully catalytic wall boundary condition was used. The cases selected from the FIRE II flight ${ }^{6}$ are listed in Table 1 . These conditions cover the range from extreme nonequilibrium to near-equilibrium ${ }^{7}$ and were chosen to ensure that the energy exchange models are assessed in widely disparate flow regimes. The temperature profiles predicted by LAURA along the stagnation line for Case $\mathrm{C}$ are presented in Fig. 1, using the baseline set of energy exchange models denoted v173. The temperature profiles for Cases A and B are shown with the label $v 173$ in Figs. 3 and 7 . In these figures, $y$ is the distance from the wall. Case $\mathrm{A}$ is in extreme thermal nonequilibrium, while Case $\mathrm{C}$ is in thermal equilibrium through about half the shock layer.

The spectral response of the radiometer windows is reported by Dingeldein. ${ }^{13}$ It is flat between 0.23 and $2 \mu \mathrm{m}$ in wavelength, falling off sharply below $0.2 \mu \mathrm{m}$ and more gradually above $2 \mu \mathrm{m}$. The design goal is reported as a flat response from 0.2 to $6 \mu \mathrm{m}$. The various reports on FIRE quote actual spectral ranges starting between 0.2 and $0.23 \mu \mathrm{m}$, and ending between 4 and $6 \mu \mathrm{m}$. Taking these variations into account, the spectral range of 0.23 to $4 \mu \mathrm{m}(0.31$ to $5.4 \mathrm{eV})$ is considered to be closest to the actual window transmission range, and is used here. Though little radiation occurs between 4 and $6 \mu \mathrm{m}$ at these conditions, the reduced spectral range is used to provide more exact comparisons. FIRE also had scanning spectral radiometers that were designed to cover the range of 0.2 to $0.6 \mu \mathrm{m}(2.1$ to $4.1 \mathrm{eV})$. 
Mechanical problems during the flight limited forward scans to $0.3-0.5575 \mu \mathrm{m}$, and backward scans to $0.6090-0.3 \mu \mathrm{m} .{ }^{8}$ The spectral resolution is quoted as $0.004 \mu \mathrm{m}$, with a root-sumsquare uncertainty in the measured spectra of \pm 23 percent. This resolution is insufficient to resolve any atomic lines.

The radiation levels obtained from the flight data are given in Tables $3-5$. These results were taken from Cauchon ${ }^{7}$ and converted from intensities to fluxes assuming a transparent plane-parallel shock to be consistent with the present calculations. Also shown is the rootsum-square uncertainty estimated in a post-flight error analysis. It should be noted that the uncertainty in the radiation level for Case A is probably higher, as the radiometers were at the lower limit of their sensitivity range for that case. The measurements show considerable scatter during the early parts of the trajectory, with a variation of about a factor of three between high and low readings for the first several seconds. The scatter decreases as the level of radiation increases. It is noteworthy that the FIRE I data acquired in this density range for a slightly higher velocity $(11.57 \mathrm{vs} 11.37 \mathrm{~km} / \mathrm{sec})$ were higher by a factor of three than the FIRE II results for both the total and spectral radiometers. The two flights thus suggest a very sensitive dependence of radiative heating on freestream velocity under these flow conditions. At the conditions of Case B, the flight-to-flight variation is about a factor of 1.6. For Case C, it is about 1.3.

\section{Results and Discussion}

All the cases in this paper were run with 64 grid cells normal to the body in the LAURA solution. This is not sufficient to resolve the shock, especially in Cases B and C. One grid-resolved solution was obtained to quantify the effect, and indicated that the radiation prediction varies only about 15 percent. This is not enough to change any of the conclusions drawn from this study. 
Table 2: Validation of Radiation Model

\begin{tabular}{ccccc}
\hline \hline Case & Time & \multicolumn{2}{c}{ Flux, W/ $\mathrm{cm}^{2}(0.31-6.2 \mathrm{eV})$} \\
\cline { 3 - 5 } & $(\mathrm{sec})$ & LORAN & NEQAIR & Deviation \\
& & & $($ Emission $)$ & (percent) \\
\hline A & 1631.0 & 2.76 & 2.47 & -11.7 \\
B & 1634.0 & 22.2 & 21.8 & -1.8 \\
C & 1637.5 & 91.5 & 83.0 & -10.2 \\
\hline \hline
\end{tabular}

\section{Radiation Model}

The initial validation of the LORAN nonequilibrium radiation model is reported in Ref. 5. To illustrate its accuracy for the FIRE II flow conditions, Table 2 compares it to the NEQAIR code of Park. ${ }^{14}$ NEQAIR is a detailed line-by-line nonequilibrium radiation model. The NEQAIR results reported here were obtained using its "emission only" mode, which sums the total emission from each radiating line without considering the line shape. This mode is fast and avoids problems of spectral resolution. The agreement between the two methods is within 12 percent and is considered excellent. (The slight differences between this table and Tables 3-5 are caused by changes made to the LAURA code between the two sets of computations and by the use of different spectral limits.)

\section{Energy Exchange Models}

The radiation predictions for the various energy exchange models are summarized and compared with the flight data in Tables 3 to 5. The predictions are broken out into atomic continuum, molecular band, and atomic line contributions. Model variations are denoted by the run identifiers defined above in the discussion of the various energy exchange models. For example, the run identifier $v 173$ implies the use of Eq. 4 for the vibrational-translational energy exchange cross section, $T_{d}=T_{t}^{\cdot 7} T_{v}^{\cdot 3}$, and the use of the average vibrational energy 
in the dissociation energy loss. Though the energy exchange processes examined are all interrelated, only one model is varied at a time from the $v 179$ model baseline. The effect of each energy exchange model and its comparison to the flight data are discussed in detail in the sections that follow.

\section{Dissociation Temperature}

As shown in the stagnation line temperature profiles, the translational temperature $T_{t}$ is much higher than $T_{v}$ near the shock. Increasing the weighting of the dissociation temperature, $T_{d}$, on $T_{t}$ in Eq. 1 therefore results in faster dissociation of the molecular species. This dissociation removes both translational and vibrational energy, decreasing $T_{t}$ and $T_{v}$. The increased dissociation is shown in Fig. 2 which compares the $N_{2}$ and $O_{2}$ profiles along the stagnation line for Case $\mathrm{A}$ for the two extreme $T_{d}$ models. The effect on the temperature profiles is shown in Fig. 3 also for Case A. The decreased temperature results in increased density and a smaller shock standoff distance. For the three FIRE II cases, placing increasing weight on the translational temperature $T_{t}$ in the definition of the dissociation temperature $T_{d}$ decreases the total radiation. Part of the decrease occurs in the molecular bands, which depend directly on the molecular concentrations. The rest of the decrease results from the lower $T_{v}$ and decreased standoff distance affecting all three radiating mechanisms. Figure 4 shows the emission profiles for the three $T_{d}$ models for Case A. The sensitivity to the $T_{d}$ model decreases at the later flight times of Cases B and C.

Tables 3-5 reveal that in all three cases the predictions are closer to the flight data when $T_{t}$ is weighted more in the definition of $T_{d}$, confirming other recent work on this model. For Cases A and B, agreement within about a factor of three is found. For Case C, the variations caused by this model are within the uncertainty of the flight data. 


\section{Vibrational-Translational Energy Exchange Cross Section}

Figures 5-6 present the effect of the different vibrational-translational energy exchange cross sections in Eqs. 4-6 on the temperature and radiative emission profiles. For a smaller $\sigma_{v}$, the relaxation time $\tau_{v}$ increases. Increasing $\tau_{v}$ delays the relaxation of translational to vibrational energy. This increases $T_{t}$ while reducing $T_{v}$, as illustrated in Fig. 5 for Case A. Although the standoff distance increases with increasing $T_{t}$, the reduction in radiation due to the lower $T_{v}$ in this model more than compensates. The lower $T_{v}$ reduces the radiation from all three mechanisms. Figure 6 shows the emission profiles for Case A. The lowest radiation for all three FIRE cases is predicted with Eq. 6, and the highest with Eq. 5. Comparing the predicted radiation for the 179, v273, and 1979 models in Tables 3-5 shows that the influence of $\sigma_{v}$ is largest in Case A.

Again all three cases are closest to FIRE II when the radiation prediction is lowest, with Eq. 6, though only Case A exhibits much sensitivity or discrimination among the models. The predictions in Case B remain high, while the effect on Case C is within the data uncertainty.

\section{Energy Exchange in Dissociation}

Figure 7 compares the temperature profiles for Eq. 9 with $c_{2}=1$ (the baseline model) and $c_{2}=2$ for Case B. The increased $\Delta E_{v}$ in the Devs model $\left(c_{2}=2\right)$ reduces $T_{v}$ in the region behind the shock where dissociation occurs, and increases it deeper in the shock layer where recombination begins. It also results in a smaller shock standoff distance. Figure 8 presents the radiative emission for Case $\mathrm{B}$ and confirms that the lower $T_{v}$ near the shock results in reduced radiation, while the higher $T_{v}$ in the layer increases it. This is observed in the tables as a decrease in the molecular band radiation and a slight increase in the atomic contributions, resulting in a net decrease in the total radiation. (The lower peak in the dashed curve of Fig. 8 is the atomic radiation peak.)

For Cases $\mathrm{A}$ and $\mathrm{B}$, the decreased radiation resulting from the devs model brings the prediction closer to the flight measurement, but it remains high. For Case $\mathrm{C}$ the effect is 
within the data uncertainty, but this is the only model where the prediction is lower than the flight measurement. Table 5 also includes a solution run with the dissociation energy exchange model of Eq. 7 using $c_{1}=0.3$ (denoted 3dis) for comparison. A flag was introduced in LAURA to suppress negative temperatures. No significant variation is observed that would encourage the use of this model.

As with the two previous energy exchange models, the sensitivity to this model decreases as the flowfield tends toward equilibrium. The decreasing sensitivity to all these models near equilibrium is expected, since they govern the exchange between energy modes in the twotemperature thermal nonequilibrium model. When dealing with flowfields near equilibrium, the choice of formulation for these models is not as important.

\section{Spectral Radiation Comparison}

To obtain further information on the validity of the energy exchange models, the FIRE spectral radiometer measurements (vs. wavelength, $\lambda$, in $\mu \mathrm{m}$ ) are compared with those ex-

change models whose predictions are closest to the flight measurements. Only continuum radiation is shown for the predicted spectra, since the resolution of the flight spectral radiometer is not sufficient to record any line radiation. In evaluating the flight spectra presented in this paper, it should be noted that only a few are available in the literature. Those that have been published are the ones that were judged to contain the least amount of noise. This criterion had the effect of selecting spectra that correspond to the lower range of flight radiation measurements. It is not known whether the unpublished data which correspond to the higher range of measurements had increased intensity levels or broader spectra or both.

Case A $(t=1631 \mathrm{sec})$

The spectral distribution of radiation has been examined for Case A for the v3\%3 solution. Figure 9(a) shows the prediction, while Fig. 9(b) is a flight spectral radiometer scan at $1631.3 \mathrm{sec}$ reproduced from Cauchon. ${ }^{8}$ While the prediction is much higher than the 
flight scan, they are qualitatively similar. Both show radiation from the $N_{2}^{+}$first negative band, whose two major band heads can be distinguished. (The spike in the flight spectrum between 0.5 and $0.6 \mu \mathrm{m}$ has been attributed to a spurious signal. ${ }^{8}$ ) Integrating the LORAN predictions over this limited spectral range results in a value that is high but within the data scatter at this flight condition.

\section{Case $B(t=1634 \mathrm{sec})$}

Figure 10 compares the 2evs result for Case B to a flight spectrum at $1634.43 \mathrm{sec}^{8}$ The qualitative agreement is comparable to that shown for Case A in Fig. 9. Integrating the predicted radiation over the range of the spectral radiometer gives a result that again, while high, is within the scatter of the flight data given in Fig. 13 of Cauchon. ${ }^{7}$ Also shown on Fig. 10 is a spectrum from a solution using the Dunn \& Kang chemical kinetics model. ${ }^{15}$ The radiation prediction for this case is included in Table 4, where it is denoted dk73. This prediction is much closer to the FIRE II measurements than any of those obtained for

Case B with the Park kinetics. ${ }^{3}$ (The same cannot be said in Case A (see Table 3).) The differences between these two chemical kinetics models are complex and should be studied further. It is interesting to note, however, that the distribution and relative magnitudes of the several band heads in this figure agree quite well with the flight data, and the Dunn \& Kang prediction exhibits quantitative as well as qualitative agreement with the measured spectrum.

\section{Case C $(t=1637.5$ sec $)$}

Figure 11 compares the predicted v373 spectrum for Case $\mathrm{C}$ to the nearest available flight spectrum, at $1636.43 \mathrm{sec}$. By this point in the trajectory, the radiometer windows are approaching their melting point. A detailed post-flight analysis determined, however, that the windows cause less than a 10 percent change in the measured radiation. ${ }^{7}$ The predicted spectrum is excessive. Because this case is close to thermal equilibrium, solutions were also 
generated assuming a single temperature, for both the Park and Dunn \& Kang chemical kinetics. These allow an assessment of the extent and impact of thermal nonequilibrium, and are included in Table 5 (v1eq and dkeq, respectively). The v1eq result is shown on Fig. 11 as the dashed line. The agreement between this spectrum and the flight spectrum is remarkable. (Recall that the spectral radiometer did not measure below $0.3 \mu \mathrm{m}$.)

\section{Cases A, B, and C}

Referring again to the temperature profiles for the three cases, it is clear that the thermal relaxation is in two stages with different time constants. Right behind the shock, $T_{t}$ decreases rapidly as a result of dissociation. The decrease in $T_{t}$ then slows indicating the involvement of other slower reactions, such as ionization. This two-stage equilibration is much less apparent for the Dunn \& Kang chemical kinetics model (see Fig. 12), and is not an issue in the equilibrium solutions. The improved agreement noted for Case B with the Dunn \& Kang solution and for Case C with the equilibrium solution suggests, therefore, that this second equilibration process deserves further study. Though the agreement in Case A (within a factor of three) has been considered good because of the uncertainties inherent at these low levels of radiation, this case also may benefit from further scrutiny of this process.

\section{Conclusions}

A study was conducted to evaluate the influence of several energy exchange models used in nonequilibrium computational fluid dynamics. Flowfield solutions obtained from LAURA with various forms of the exchange models were used as input to LORAN, a recently developed nonequilibrium radiation model. The results were evaluated by comparison to the Project FIRE II flight data taken at 1631, 1634, and $1637.5 \mathrm{sec}$ in the early part of the trajectory.

Models for the rate controlling temperature for dissociation, $T_{d}$, vibrational-translational energy exchange cross section, $\sigma_{v}$, and energy exchange in dissociation, $\Delta E_{v}$, were considered. 
Formulations that increased $T_{d}$, reduced $\sigma_{v}$, or increased $\Delta E_{v}$ resulted in reduced radiation. The greatest effect was seen at 1631 sec when nonequilibrium was most extreme. Radiative heating predictions from the different formulations varied more than 100 percent for this case. The lower radiation predictions generally fell closer to the flight measurements.

In addition to examining the total radiation, predicted radiation spectra were compared with the flight data. These indicated that the close agreement in total radiation was fortuitous in some cases because there was excessive energy in the molecular bands. At $1637.5 \mathrm{sec}$, a thermal equilibrium result provided better spectral agreement. At $1634 \mathrm{sec}$, an alternate chemical kinetics set improved the spectral comparison. These results suggest that chemical kinetic effects deserve further attention.

This study has demonstrated that the examination of radiative heating measurements from flight experiments can provide a means of evaluating models used in computational fluid dynamics (CFD) for nonequilibrium flowfields. Further study of the FIRE radiometer data and results of future flight experiments can be expected to provide important tests of such models leading to improvements in CFD solutions.

\section{References}

${ }^{1}$ Gnoffo, P. A., “Upwind-Biased Point-Implicit Relaxation Strategies for Viscous Hypersonic Flows," AIAA Paper 89-1972, June 1989.

${ }^{2}$ Richardson, N. R., "Project FIRE Instrumentation for Radiative Heating and Related Measurements," NASA TN D-3646, 1966.

${ }^{3}$ Park, C., “Assessment of Two-Temperature Kinetic Model for Ionizing Air," Journal of Thermophysics and Heat Transfer, Vol. 3, No. 3, July 1989, pp. 233-244.

${ }^{4}$ Gnoffo, P. A., Gupta, R. N., and Shinn, J. L., "Conservation Equations and Physical Models for Hypersonic Air Flows in Thermal and Chemical Nonequilibrium," NASA TP 2867, Feb. 1989. 
${ }^{5}$ Hartung, L. C., Nonequilibrium Radiative Heating Prediction Method for Aeroassist Flowfields with Coupling to Flowfield Solvers, PhD Thesis, North Carolina State University, Raleigh, 1991.

${ }^{6}$ Lewis, J. H., Jr. and Scallion, W. I., "Flight Parameters and Vehicle Performance for Project FIRE Flight II, Launched May 22, 1965,", NASA TN D-3569, Aug. 1966.

${ }^{7}$ Cauchon, D. L., "Radiative Heating Results from the FIRE II Flight Experiment at a Reentry Velocity of 11.4 Kilometers per Second,", NASA TM X-1402, July 1967.

${ }^{8}$ Cauchon, D. L., McKee, C. W., and Cornette, E. S., “Spectral Measurements of GasCap Radiation During Project FIRE Flight Experiments at Reentry Velocities Near 11.4 Kilometers per Second," NASA TM X-1389, Oct. 1967.

${ }^{9}$ Sharma, S. P., Huo, W. M., and Park, C., "The Rate Parameters for Coupled VibrationDissociation in a Generalized SSH Approximation - Schwartz, Slawsky, and Herzfeld," AIAA Paper 88-2714, June 1988.

${ }^{10}$ Millikan, R. C., and White, D. R., "Systematics of Vibrational Relaxation," Journal of Chemical Physics, Vol. 39, No. 12, Dec. 1963, pp. 3209-3213.

${ }^{11}$ Park, C., "Problems of Rate Chemistry in the Flight Regimes of Aeroassisted Orbital Transfer Vehicles," Progress in Astronautics and Aeronautics: Thermal Design of Aeroassisted Orbital Transfer Vehicles, Vol. 96, AIAA, 1985, pp. 511-537.

${ }^{12}$ Park, C., "Two-Temperature Interpretation of Dissociation Rate Data for $\mathrm{N}_{2}$ and $\mathrm{O}_{2}$, " AIAA Paper 88-0458, Jan. 1988.

${ }^{13}$ Dingeldein, R. C., "Radiative and Total Heating Rates Obtained from Project FIRE Reentries at 37,000 Feet per Second," Paper presented at the AFFDL/ASSET Advanced Lifting Reentry Technology Symposium, Miami Beach, Florida, Dec. 1965.

${ }^{14}$ Park, C., "Nonequilibrium Air Radiation (NEQAIR) Program: User's Manual," NASA TM-86707, July 1985.

${ }^{15}$ Dunn, M. G., and Kang, S., "Theoretical and Experimental Studies of Reentry Plasmas," NASA CR 2232, 1973. 


\section{Figure Captions}

1. Temperature Profiles for FIRE II - Case C

2. Effect of $T_{d}$ Models on Molecular Dissociation - Case A

3. Effect of $T_{d}$ Models on Temperature Profiles - Case A

4. Effect of $T_{d}$ Models on Radiative Emission Profiles - Case A

5. Effect of $\sigma_{v}$ Models on Temperature Profiles - Case A

6. Effect of $\sigma_{v}$ Models on Radiative Emission Profiles - Case A

7. Effect of Molecular Dissociation Models on Temperature Profiles - Case B

8. Effect of Molecular Dissociation Models on Radiative Emission Profiles - Case B

9.: a) Predicted Radiation Spectrum - Case A

b) Measured Radiation Spectrum - $1631.3 \mathrm{sec}$

10.: a) Predicted Radiation Spectra - Case B

b) Measured Radiation Spectrum - $1634.43 \mathrm{sec}$

11.: a) Predicted Radiation Spectra - Case C

b) Measured Radiation Spectrum - $1636.43 \mathrm{sec}$

12. Effect of Chemical Kinetics Model on Temperature Profiles - Case B 
Table 3: Effect of Energy Exchange Models in Case A

\begin{tabular}{ccccc}
\hline \hline \multirow{2}{*}{ Run } & \multicolumn{4}{c}{ Flux, W/cm $\mathrm{cm}^{2}(0.31-5.4 \mathrm{eV})$} \\
\cline { 2 - 5 } Identifier & Atomic & Mol. & Atomic & Total \\
& Cont. & Band & Line & \\
\hline flight & & & & $.16 \pm 20 \%$ \\
v110 & $.1 \mathrm{E}-4$ & .54 & $.4 \mathrm{E}-3$ & .54 \\
v155 & $.7 \mathrm{E}-5$ & 2.0 & $.5 \mathrm{E}-2$ & 2.0 \\
v173 & $.1 \mathrm{E}-4$ & 1.1 & $.9 \mathrm{E}-3$ & 1.1 \\
v273 & $.1 \mathrm{E}-4$ & 3.2 & $.2 \mathrm{E}-1$ & 3.2 \\
v373 & $.1 \mathrm{E}-4$ & .41 & $.5 \mathrm{E}-3$ & .41 \\
dk73 & $.2 \mathrm{E}-3$ & 1.7 & $.5 \mathrm{E}-1$ & 1.7 \\
v1732evs & $.1 \mathrm{E}-4$ & .72 & $.5 \mathrm{E}-3$ & .73 \\
\hline \hline
\end{tabular}

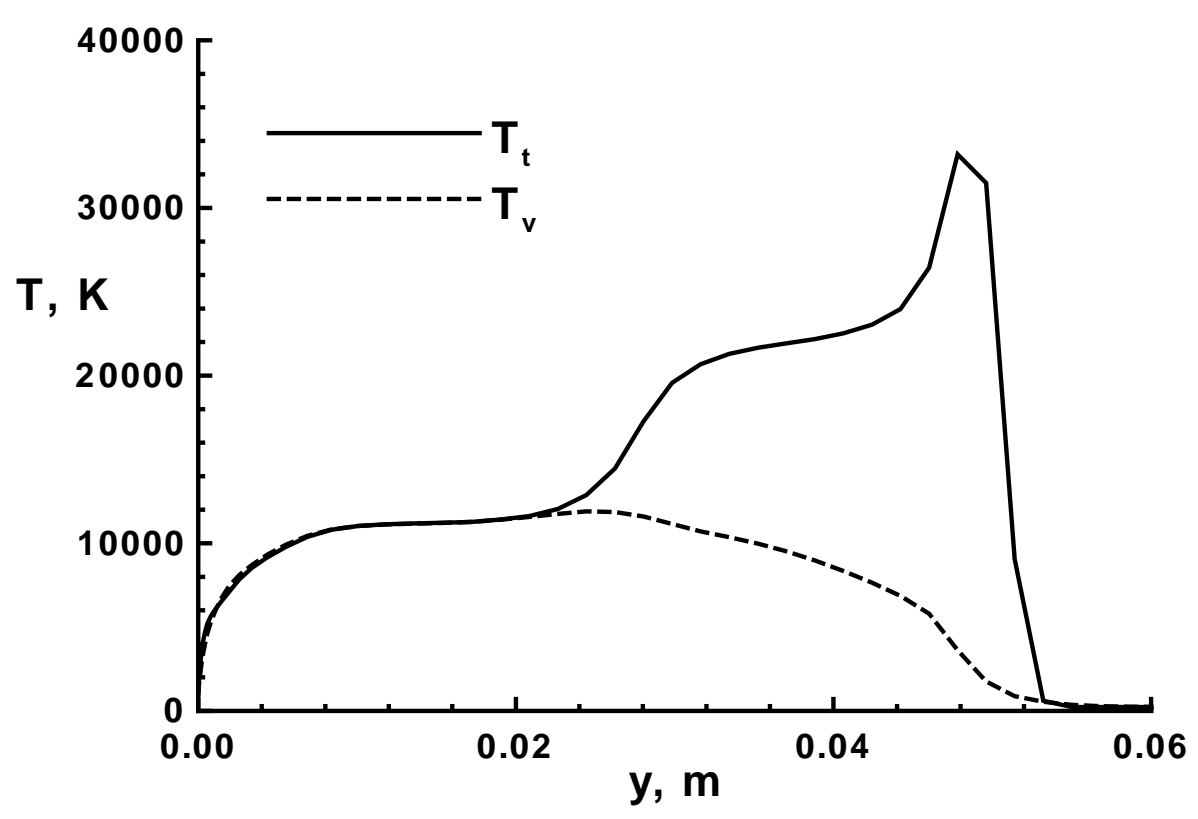

Figure 1: Temperature Profiles for FIRE II - Case C 
Table 4: Effect of Energy Exchange Models in Case B

\begin{tabular}{ccccc}
\hline \hline \multirow{2}{*}{ Run } & \multicolumn{4}{c}{ Flux, W/cm $\mathrm{cm}^{2}(0.31-5.4 \mathrm{eV})$} \\
\cline { 2 - 5 } Identifier & Atomic & Mol. & Atomic & Total \\
& Cont. & Band & Line & \\
\hline flight & & & & $8.2 \pm 20 \%$ \\
v110 & $.2 \mathrm{E}-1$ & 17.7 & 2.7 & 20.3 \\
v155 & $.4 \mathrm{E}-1$ & 19.1 & 3.8 & 22.9 \\
v173 & $.3 \mathrm{E}-1$ & 17.7 & 3.3 & 21.1 \\
v273 & $.7 \mathrm{E}-1$ & 22.5 & 5.2 & 27.8 \\
v373 & $.1 \mathrm{E}-1$ & 17.6 & 2.2 & 19.9 \\
d1k73 & .3 & 3.1 & 7.9 & 11.4 \\
v1732evs & .1 & 12.1 & 6.0 & 18.3 \\
\hline \hline
\end{tabular}

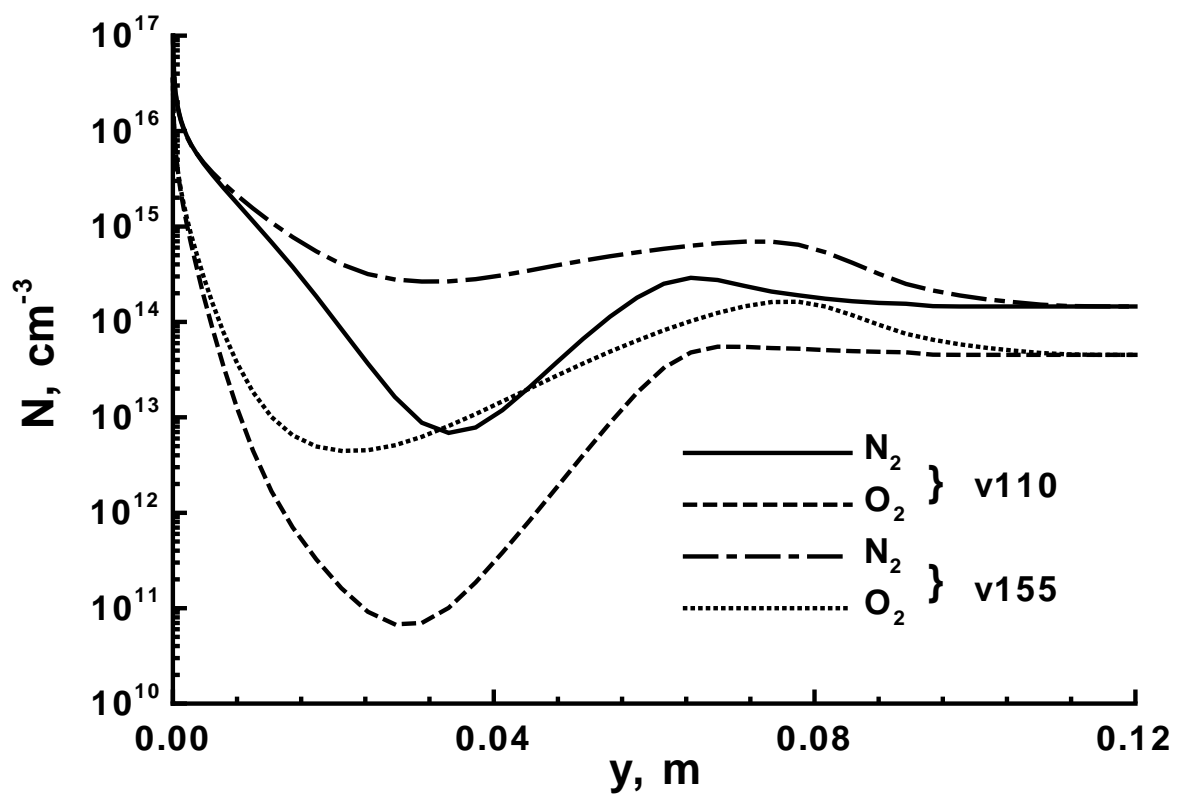

Figure 2: Effect of $T_{d}$ Models on Molecular Dissociation - Case A 
Table 5: Effect of Energy Exchange Models in Case C

\begin{tabular}{ccccc}
\hline \hline Run & \multicolumn{3}{c}{ Flux, W/ $\mathrm{cm}^{2}(0.31-5.4 \mathrm{eV})$} \\
\cline { 2 - 5 } Identifier & Atomic & Mol. & Atomic & Total \\
& Cont. & Band & Line & \\
\hline flight & & & & $81.7 \pm 20 \%$ \\
v110 & 3.8 & 33.5 & 45.3 & 82.7 \\
v155 & 3.9 & 38.9 & 46.2 & 89.0 \\
v173 & 3.9 & 35.4 & 45.7 & 84.9 \\
v273 & 4.1 & 38.8 & 49.2 & 92.1 \\
v373 & 3.8 & 33.6 & 44.5 & 81.9 \\
dkeq & 5.2 & 18.8 & 99.9 & 123.9 \\
v1eq & 6.5 & 5.8 & 75.6 & 87.8 \\
v1732evs & 4.6 & 20.8 & 52.0 & 77.4 \\
v1733dis & 4.6 & 15.5 & 52.6 & 72.8 \\
\hline \hline
\end{tabular}




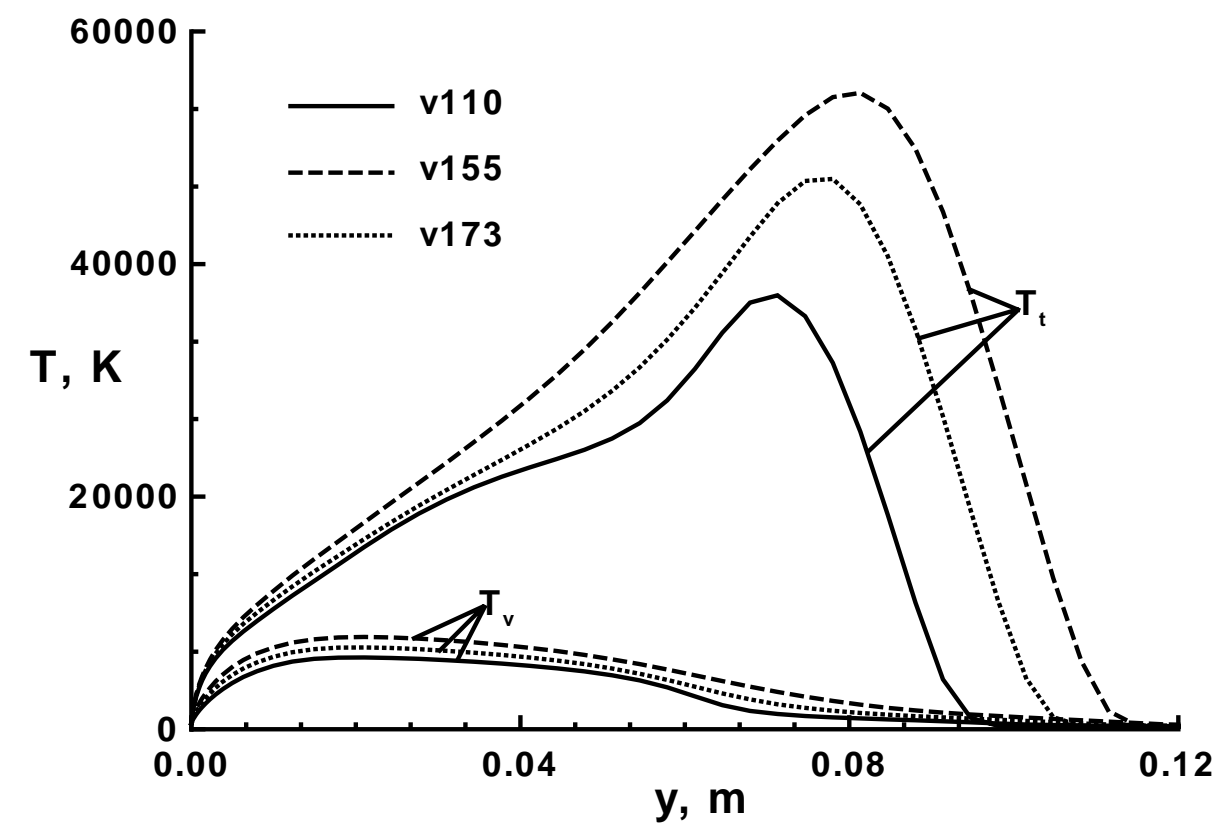

Figure 3: Effect of $T_{d}$ Models on Temperature Profiles - Case A

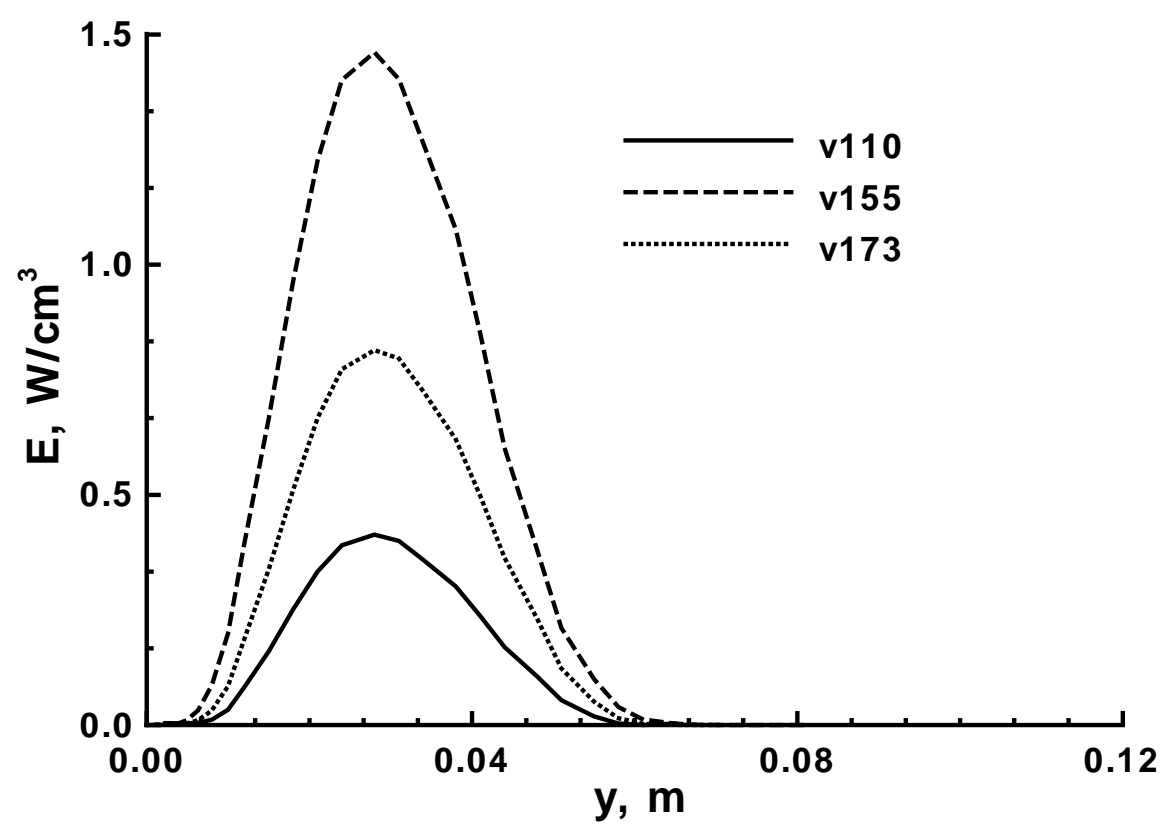

Figure 4: Effect of $T_{d}$ Models on Radiative Emission Profiles - Case A 


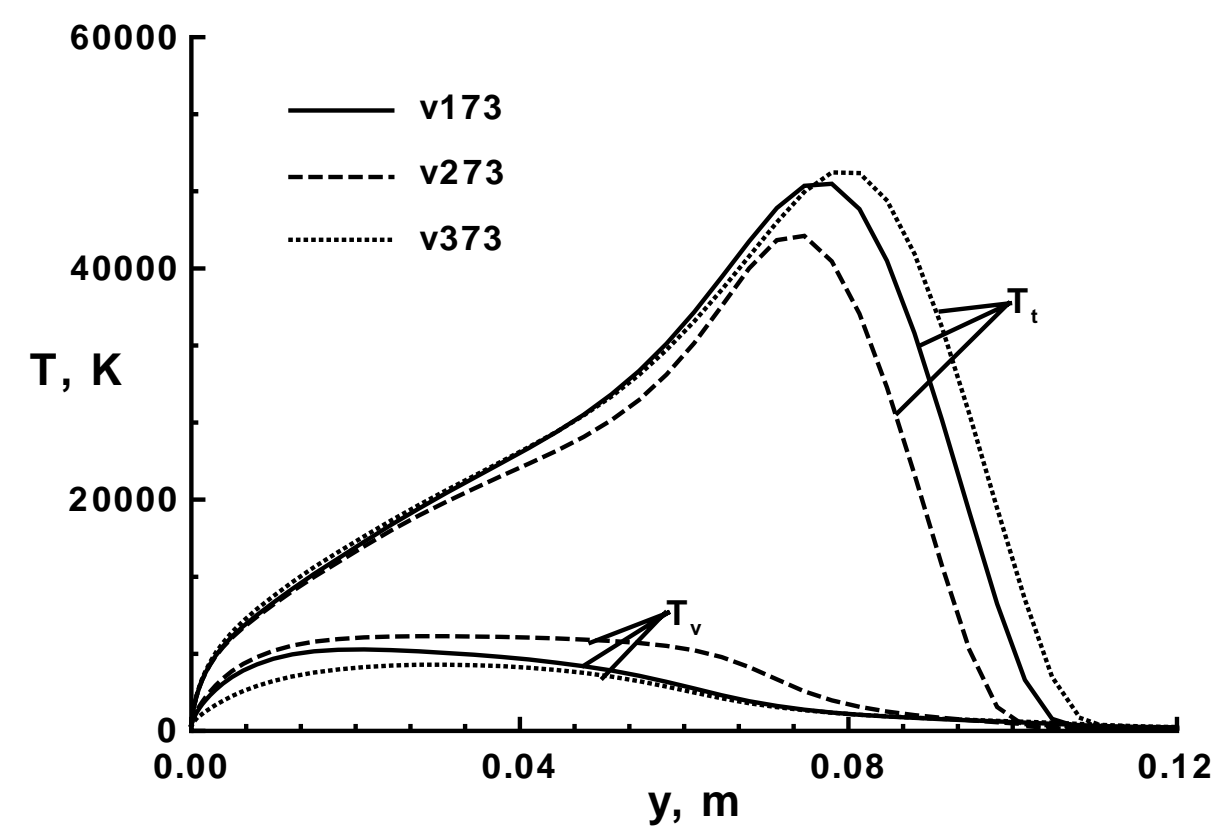

Figure 5: Effect of $\sigma_{v}$ Models on Temperature Profiles - Case A

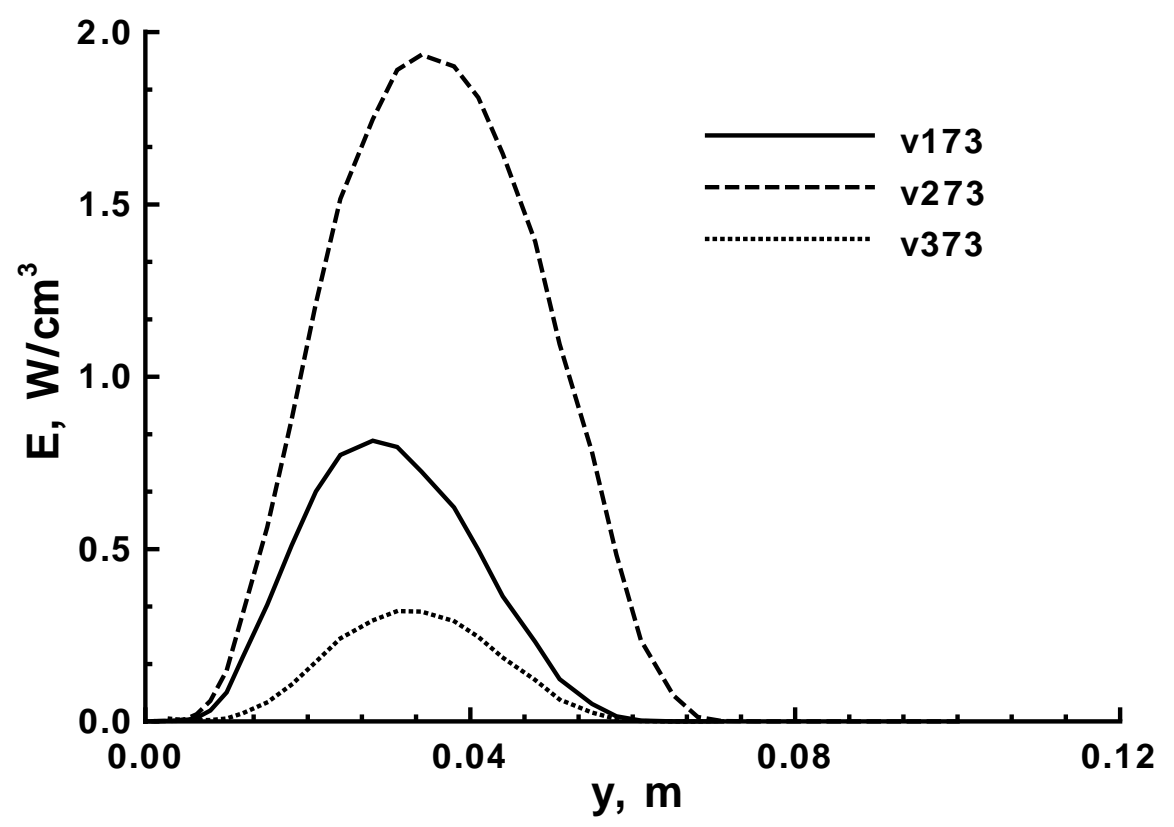

Figure 6: Effect of $\sigma_{v}$ Models on Radiative Emission Profiles - Case A 


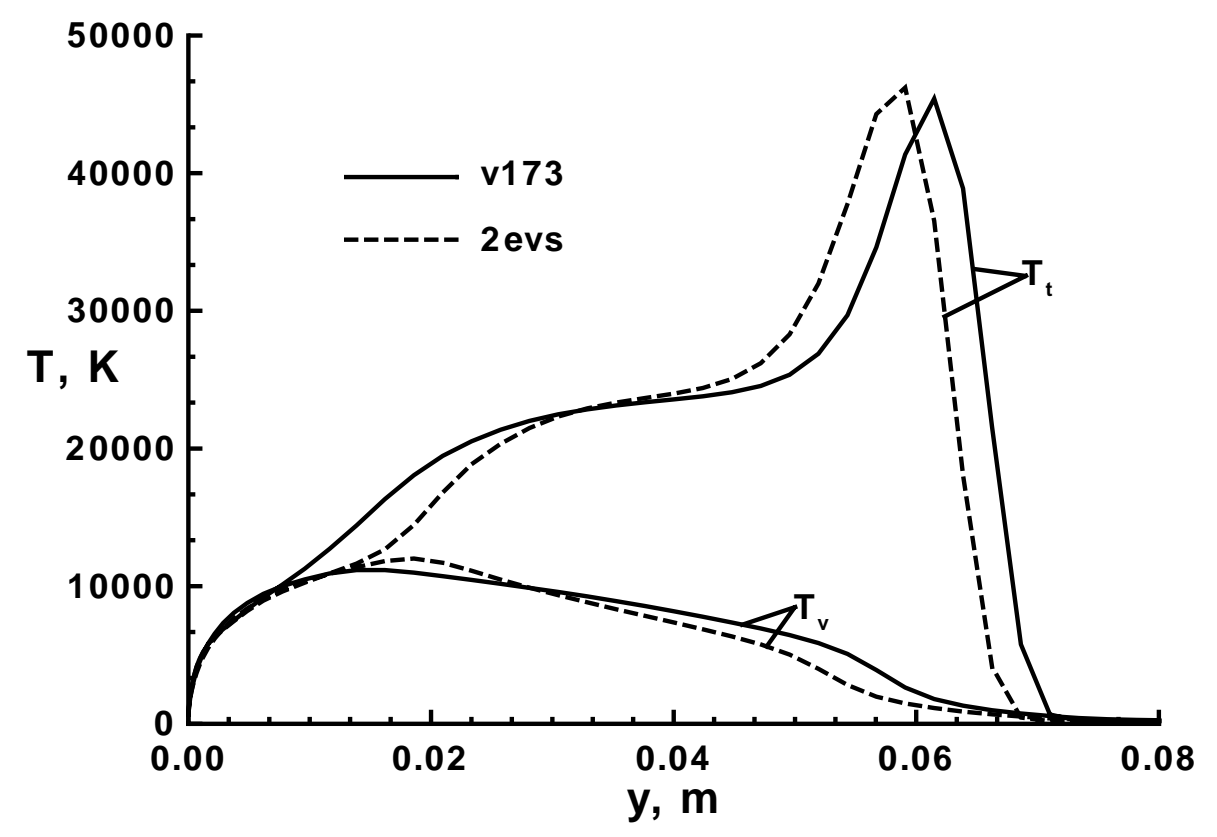

Figure 7: Effect of Molecular Dissociation Models on Temperature Profiles - Case B

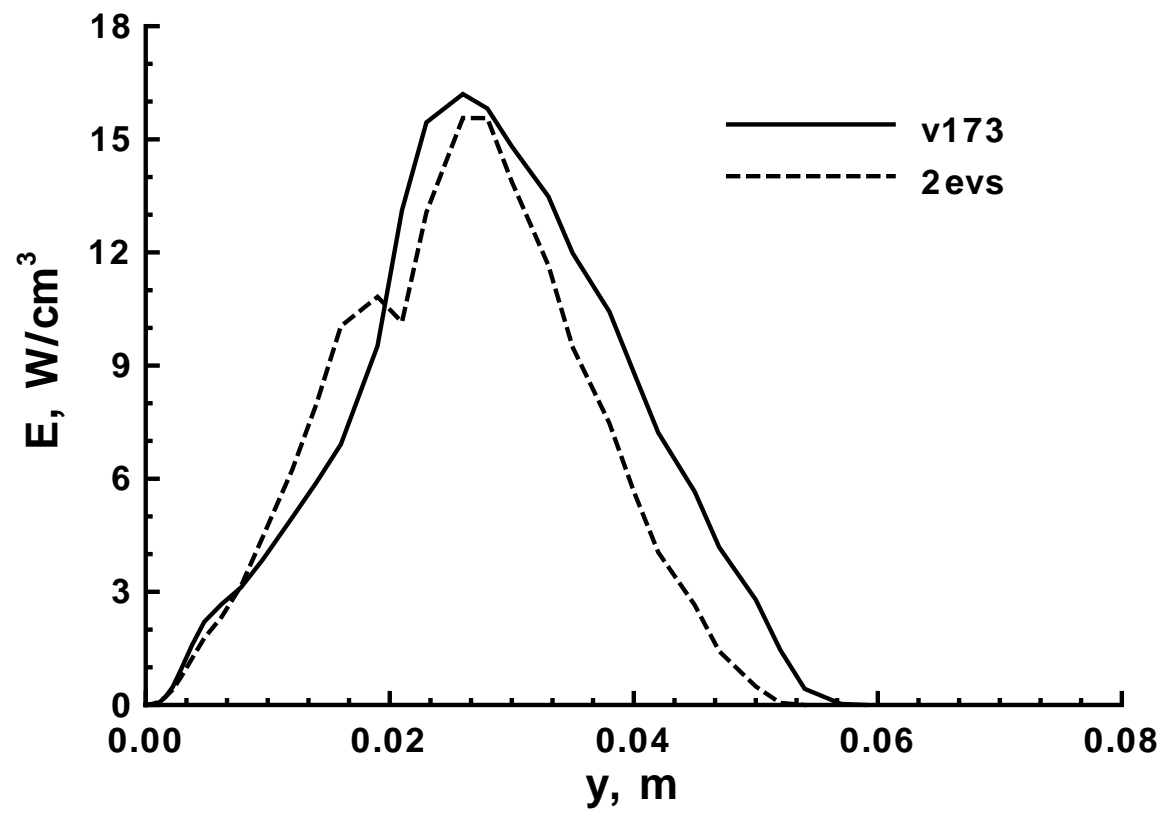

Figure 8: Effect of Molecular Dissociation Models on Radiative Emission Profiles - Case B 


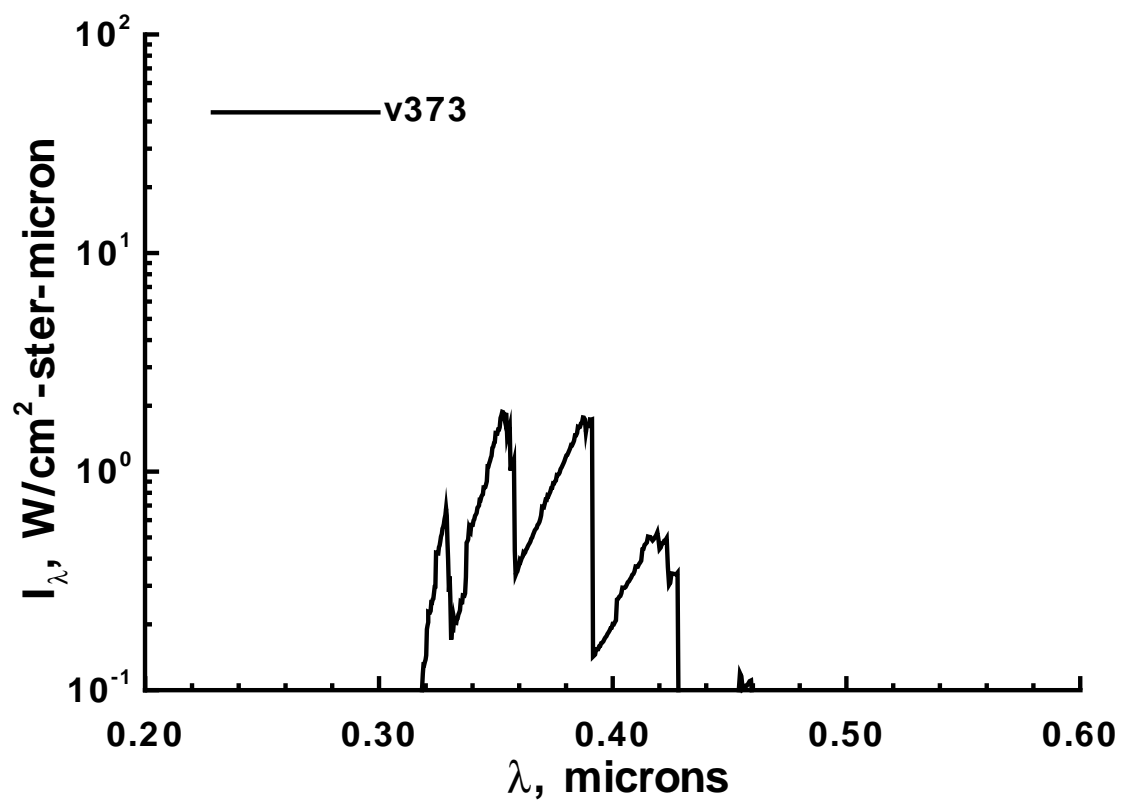

Figure 9: a) Predicted Radiation Spectra - Case A

Figure 9: b) Measured Radiation Spectrum - $1631.3 \mathrm{sec}$ 


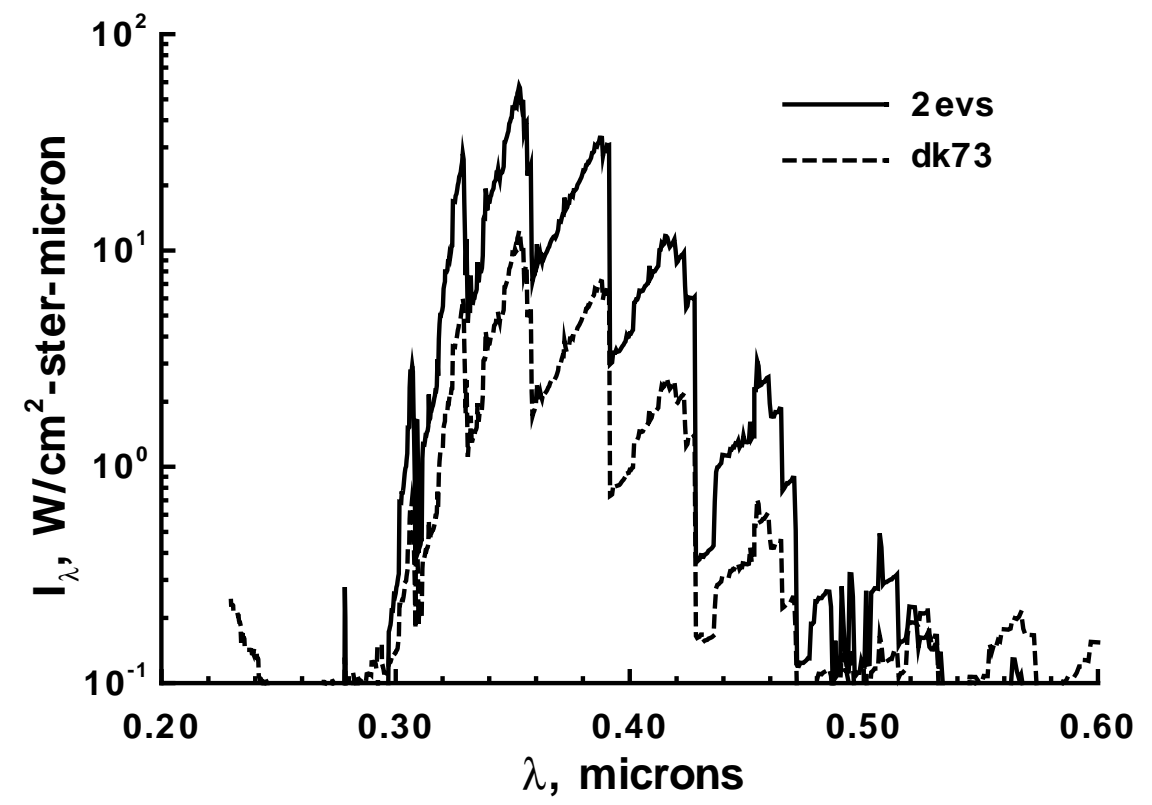

Figure 10: a) Predicted Radiation Spectra - Case B

Figure 10: b) Measured Radiation Spectrum - $1634.43 \mathrm{sec}$ 


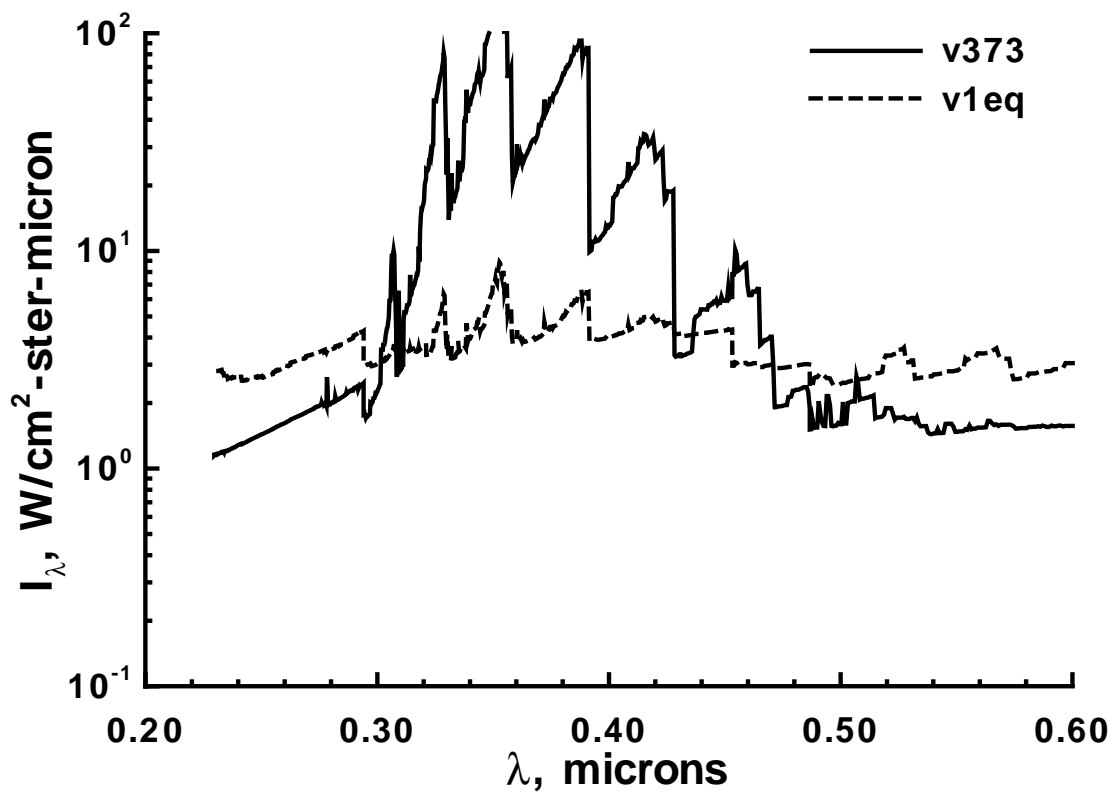

Figure 11: a) Predicted Radiation Spectra - Case C

Figure 11: b) Measured Radiation Spectrum - $1636.43 \mathrm{sec}$ 


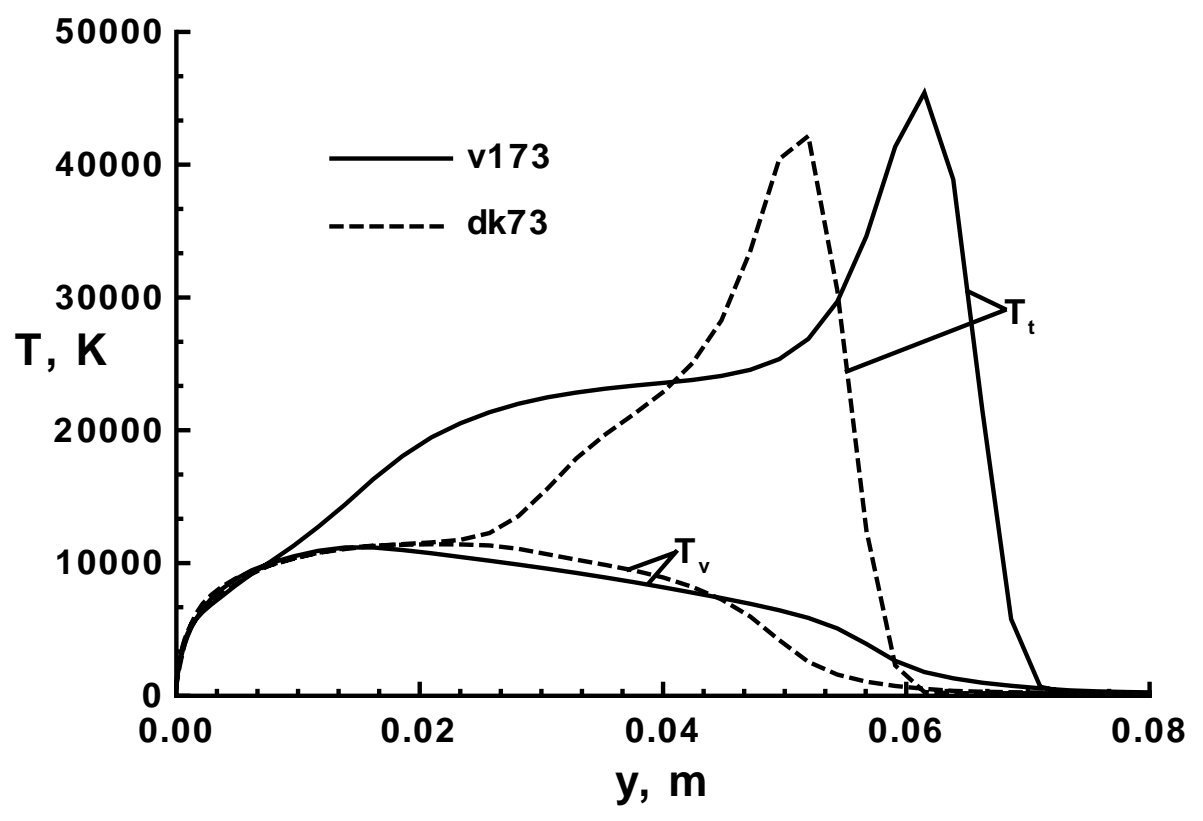

Figure 12: Effect of Chemical Kinetics Model on Temperature Profiles - Case B 